\title{
Amino Acids in Pathological Gambling and in its Alleviation: Pharmacological Approaches
}

\author{
R.C. Gupta*
}

SASRD, Nagaland University, Medziphema 797 106, India

\begin{abstract}
Labelling Pathological gambling (PG) with a tag of Socio-Psychological problem more than medical causes hampers its pharmacological treatment. Because of this, its neurobiology has been studied only a little, limited to abnormalities in the serotonergic, noradrenergic and dopaminergic systems. Besides this; studies on the role of precursors of these neurotransmitters are almost negligible. Higher concentrations of known precursors like tryptophan, tyrosine and phenyl alanine have been reported in the CSF of pathological gamblers. In addition to this, recently many other inhibitory and excitatory amino acids like GABA, taurine, aspartic and glutamic acids have been found to be in much higher concentration, providing a basis for the candidate amino acids in the therapy of PG. Many of these amino acids have beneficial actions in a number of CNS problems. On this basis, as most of these amino acids are non-toxic and natural, such amino acids can be used as supplements /compliments in PG therapy. One of these amino acids, taurine, a sulfur containing amino acid which is now part of various energy drinks, anti-ageing and anti-diabetes formulas, mood enhancers and many more alternative medicine applications, also could exert beneficial actions in alleviating PG. Therapy with amino acids also has its own limitations; hence development of pro-drug derivatives of these amino acids may constitute a new class of therapeutic agent for PG. This review is a blended form of synthesis and synopsis about various amino acids in the context of PG, considering their potential utility and providing a provocative stimulus for scientific thinkers.
\end{abstract}

Keywords: Pathological gambling (PG), pharmacotherapy, amino acids, taurine, analogues and derivatives.

\section{INTRODUCTION}

Perhaps gambling is as old as our planet. It is very hard to trace the history of gambling but it prevails through all the ages. Gambling can be defined in various ways. One of the definitions can be: gambling is an activity in which value is placed upon a game or event or a bet of any type that has no definite outcome and to a certain extent the result is chance dependent. Statistics reveal that a sizeable portion of our society engages in activities that are included under the definition of gambling. In the process of gambling it is observed that certain individuals become habituated to it, and develop certain characteristics which put gambling at first place in their motivational priorities. Various names have been coined to depict such forms of gambling, such as compulsive gambling or excessive gambling; later this complex nature of gambling has been given an additional name, pathological gambling (PG). Technically pathological gambling is not a disease but a impulse control disorder [1], progressive in nature and chronic. Some of the other phenomena classified as impulse control disorders include attention deficit, hyper activity disorder, obsessive compulsive disorder and substance abuse [2, 3].

A number of studies have assessed the prevalence of PG, which ranges between 1.2 to $1.5 \%$ of the adult population [4]. It is more common in urban areas, poor areas, or areas

*Address correspondence to this author at the SASRD, Nagaland University, Medziphema 797 106, India; Tel: 913862247102 ; Fax: 913862247113 ;

E-mails: rameshgupta1954@yahoo.com, rameshgupta1954@gmail.com where there is higher availability of gambling opportunities. Such studies also suggest that male adolescents, ethnic minorities, and psychiatric patients are more risk prone [5]. The causes of PG likely have several potential contributions; however psychologists give more importance to individual factors. Apart from these theories, there is another consideration, linking $\mathrm{PG}$ to brain chemical imbalance thereby providing a role for neurotransmitters, which are responsible for creating different physiological conditions. Currently such known neurotransmitters are noradrenalin, serotonin and dopamine. Their role in PG are more or less now established. Besides neurogenic amines there are several amino acids in brain which also play an important role possibly as neurotransmitters or neuro-modulators. Many of such amino acids have altered concentrations in the cerebrospinal fluid (CSF) of pathological gamblers. Some of the important amino acids are tryptophan, phenylalanine, tyrosine, aspartic acid, glutamic acid, orthinine, GABA and arginine. Besides these, several branch chain amino acids, glycine, and sulfur-containing amino acids like, methionine and taurine also have been implicated [6-8]. Some of these amino acids are also in use as psychotherapeutic agents as well as in correcting various CNS disorders. An amino acid like GABA modulates and mediates dopaminergic functions. Tryptophan which is a precursor of serotonin also has its own effects, and its deficiency is associated with encounter mood lowering, memory impairment and increased aggression. Arginine has a role in patho-physiology of Huntington's disease. The amino acids phenylalanine and tyrosine, besides being precursors of noradrenalin and dopamine, have their own effects on mood and other 
cognitive events. Similarly glutamic acid may have promising treatment possibilities in psychiatry and neurology. Sulfur-containing amino acids play special roles in structure and function of brain, and they provide a portion of brain Sulfur also. Methonine, cystein and taurine have a long list of potential actions in the CNS [9]. In PG too there is alternation in their concentrations. Taurine which is synthesized from cystein has a wide spectrum of central actions, from anticonvulsant to anti-aggressive and anti tremor to many more [10-14].

In general pathological gamblers show a peculiar nature combined with depression, psychopathic deviance, dependency /dominance, social introversion, ego strength, anxiety or even aggressiveness. All of these traits are also linked to CNS actions. Since the majority of the mentioned amino acids in one way or another exert beneficial actions in controlling such disorders, it will be logical to examine these agents in depth to ascertain their roles in PG and possibly their utility in alleviating PG.

\section{EPIDEMIOLOGY OF PATHOLOGICAL GAMBLING}

Gambling is a universal event. Studies show about 70-90 $\%$ of adults gamble some time in their life [15]; especially among adolescents it is becoming a common feature [16]. However, there are controversies about the prevalence of Pathological gambling which differ from 1 to $2 \%$ [17]; to 1.5 to $11.2 \%$ of the adult population [18]. Higher PG rates have been found among psychiatry patients, as high as 6.7 to $12 \%$ [19]. A big rise in PG has been recorded among substance abusers. PG has been linked to a history of child hood experiences of gambling in the family, high rates of stress problems and serious psychiatric conditions [20,21].

Epidemiological studies suggest especially in males that adolescence is a more vulnerable time, and the majority of future PGS is set in at this stage. A review on adolescents in the United States of America found a prevalence rate between 4.4 to $7.4 \%$ with a possibility for an additional 10 to $14 \%$ at risk for developing $P G$ [22, 23]. Besides adolescents, ethnic minorities and patients with psychiatric co-morbidities are also vulnerable [24]. Gender-wise though more males than females are Pathological gamblers, it is also possible that female PGS are not so extensively studied; yet many studies count their number as about $1 / 3$ of male PGS. Further studies on female PGS; in many cases, show linkage to their PG parents [25]. It is believed that male and female PGS have different reasons and motivations for PG, but in males it is more to get desired results while in females it is more to escape the situation, loneliness, isolation, depression, neglect and many others situations.

\section{PHENOMENOLOGY OF PATHOLOGICAL GAMBLING}

Pathological gambling is linked to a series of events which include impulsive control disorder, elevated impulsive scores on impulsiveness scales and personality profiles [26]. In fact impulsivity is a human centric activity; it is a type of unintentional behaviour by which one generally acts spontaneously without forethought. Impulsivity is an important component in PG [27]. Many studies have found that Pathological gamblers score higher on impulsivity [28]. A number of events like anti-social behaviour, personality disorder; attention deficit hyperactive disorder (ADHD) are some of the syndromes associated with PG. A high rate of suicide [29, 30], increased rates of co-morbid substance abuse and dependence [31] are also part of the personality profiles [26]. Some of the other related behaviours are neuroticism, psychosis and extraversion. Impulsivity is also related to psychoses whereas neurotic aspects of PG are linked to depression. In general pathological gamblers are possibly more extraverted than non pathological gamblers. All these supporting evidences contribute towards the conclusion that pathological gamblers are at high risk for committing repeated crime to maintain their gambling habit. Besides impulsivity there is a phenomenological link to compulsive events also, like increased rate of obsessive compulsive disorder $[31,32]$, increased score on the harm avoidance scale [33], tendency for repeating the behaviour [32], high degree of tension before exhibition of the compulsive behaviour [32], and feeling relaxed after completion of the compulsive behaviour.

\section{PHYSIOLOGY OF PATHOLOGICAL GAMBLING}

It is disheartening not to see good literature on physiological aspects of PG. From the physiological point of view $P G$ can be placed in such groups which have dysfunctional attention mechanisms similar to those for alcoholism and ADHA [34]. Pathological gamblers are more likely to repeat childhood behaviour. In PG Neurophysiological tests have shown frontal lobe lesions with hyper metabolism [35], very similar to that observed in children with ADHA. In term of heart rate PGS have increased arousal [36]. In pathological gamblers mood plays a very vital role and any disturbance in mood will have definite effects on parameters of PG. It is believed that plasma endorphins are involved in the mood disturbances [37]. Another study showed a link of increased uric acid with psychological activation during the course of gambling. However all these physiological observations do not have strong experimental data to support the claims.

\section{NEUROBIOLOGY OF PATHOLOGICAL GAMBLING}

The literature does not provide much on neuro-biology of PG. Very few neurological investigations have been recorded to assess the relationship between $P G$ and brain functioning. Impulsivity which has a definite role in $P G$ is basically because of the neurological alteration; hence assessment of a co-relation between pathological gambling and constructs of impulsivity will provide leads to formulate therapy. The inside story of the neurobiological mechanisms in etiology, pathogenesis and development of PG has implicated a role of neurotransmitters [38]. Such neurotransmitters are generally grouped in to three, serotonergic, noradrenergic and dopaminergic, of which serotonergic has a major role. Still now, these represent the core of theoretical and structural models for neurobiological occurrences and to provide corrective measures.

\section{MOLECULAR GENETICS OF PATHOLOGICAL GAMBLING}

Genes in essence provide the first contribution to the addiction process, as they produce fundamental vulnerabilities that displace normal behavioral processes. 
Recent research combining molecular genetics, endocrinology, and neuroimaging, demonstrated that variations in dopamine-related genes and hormone levels affect the physiological properties of the dopaminergic system in non-human primates, and modulate the processing of reward and social information in humans. These findings are important because they indicate the neural influence of genes conferring vulnerability to develop neuropathologies, such as pathological gambling. Molecular genetic techniques have been used to investigate the role of genetic factors in PG. Molecular genetic research has identified specific allele variants of candidate genes corresponding to the neurotransmitter systems associated with PG. Associations have been reported between pathological gamblers and allele variants of polymorphisms at dopamine receptor genes, the serotonin transporter gene, and the monoamine-oxidase A gene, although preliminary data suggest that some of these differences are gender-specific. In the recent past with the isolation of genes and genetic variants (allelepolymorphisms) between case and control groups; molecular genetics has been linked to the behavior; and addiction and its complexity in such situations are well suited to characterizing pathological gambling also [39]. Genetic association studies represent the most valid approaches for psychiatric genetic investigations. Thus there is no doubt that genetic studies are now one of the most powerful study designs; an "inside" investigation when done with proper controls can provide definitive clues for understanding the neurobiological aspects of the disorder.

\section{Conventional Molecules: Serotonin, Noradrenalin and Dopamine}

CSF is regarded as the internal milieu of the brain. CSF serves as a modified lymphatic system for the brain and spinal cord and acts as a reflection of cerebral metabolism. In fact cerebral metabolism is basically a differentiation of the gradients of metabolites that exist between ventricular (VCSF) and lumber (LCSF) cerebral fluid. LCSF reflects cerebral function, and any significant biochemical alteration will affect cerebral metabolism. Metabolism of the neurotransmitters is of great interest; in general the biogenic amines have their own distribution patters in brain and spinal cord.

\section{Serotonergic Interaction}

Of all the biogenic amines, neurons containing serotonin (5HT) are more common. Serotonin is synthesised from the amino acid tryptophan through the intermediate 5-Hydroxy Tryptophan (5-HTP), which is de-carboxylated to 5-Hydroxy Tryptamine commonly known as 5 -HT or Serotonin. 5 HT is further degraded via methylation through S-adenosyl methionine providing $\mathrm{N}-\mathrm{Me}-5-\mathrm{HT}$; or possibly $5 \mathrm{HT}$ may be metabolized by MAO to produce initially 5 -Hydroxy Indole aldehyde and finally 5-Hydroxy Indole acetic Acid (5HIAA) (Fig. 1).

Dysfunction of the serotonin system is identified as playing an important role in impulsive behaviours [40]. It is believed that the serotonin transporter gene could be involved in the pathogenesis of PG [41]. Platelet monoamine oxidase (MAO) activity has been regarded as a peripheral marker of serotonergic activity. In PG patients MAO-B activity has been found to be low [42]; thus altered serotonergic function is expected. A mechanistic insight on serotonergic function has shown a relationship between reduced CSF 5-HIAA, a metabolite of $5 \mathrm{HT}$, and varieties of impulsive/|aggressive disorder \& behaviour [42]. In a recent study on CSF monoamine patterns in PGS and control [8, 43], lower CSF levels of tryptophan and 5HT have been recorded in PGS, but opposite for 5-HIAA. The lower 5 HT and higher 5-HIAA levels may indicate an increased biotransformation of 5-HT. It is interesting to note that 5 -

\section{Trytophan}

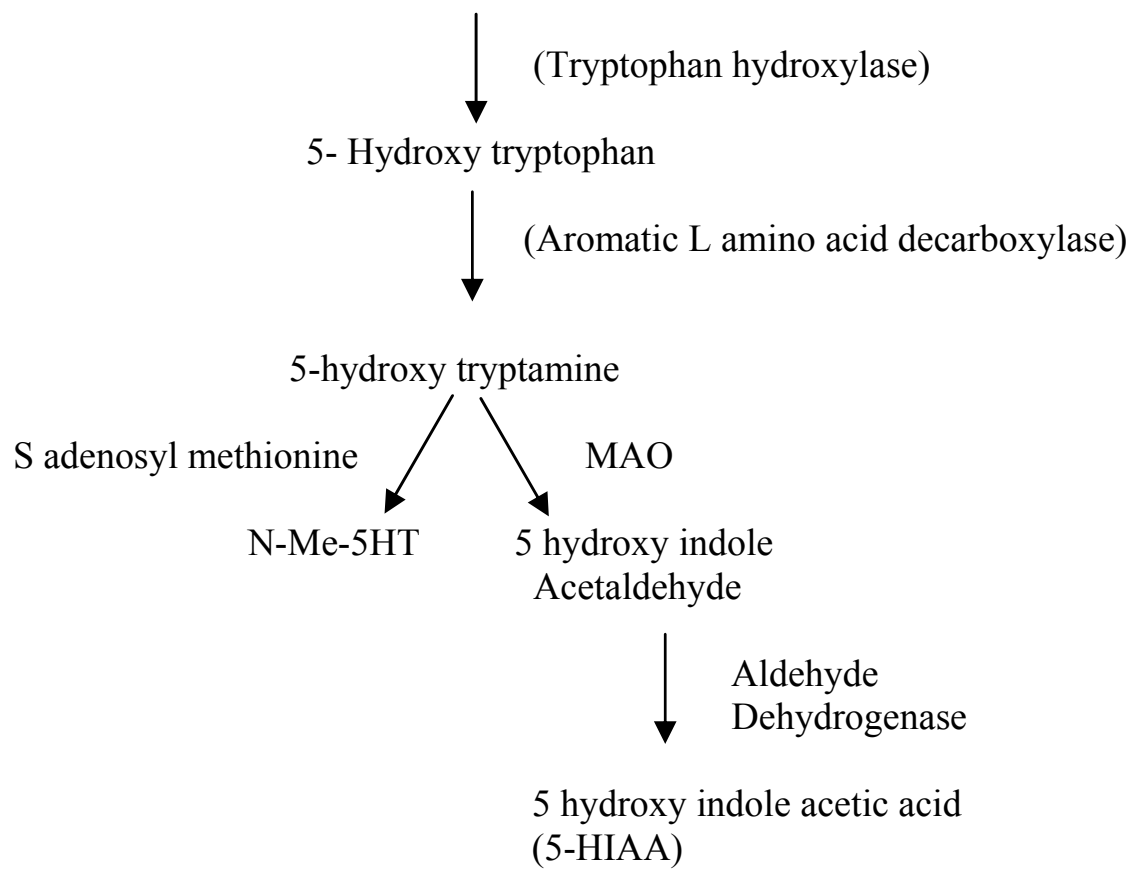

Fig. (1). Regulation of serotonin. 
HIAA concentration in CSF has been influence by intraspinal pressure $[6,44]$

\section{Noradrenergic Interaction (NA)}

Spinal cord neurons also contain significant amounts of NA. Noradrenalin is synthesised from the amino acid phenylalanine which can further degrade to vanillyl mandelic acid (VMA) or methoxy hydroxyl phenyl glycol (MHPG) depending upon involvement of MAO or COMT (Fig. 2). Noradrenalin has a role in arousal and impulse control. Studies have found higher level of metabolites of noradrenalin [45, 46]; especially MHPG is very high in concentration. The level of noradrenalin and its metabolite VMA are found to elevated in Pathological gamblers [46]. An elevated growth hormone peak in response to clonidine treatment has been also observed [47]. Since the noradrenalin system is associated with arousal activity and arousal has an important role in PG, thus it would be logical to think that noradrenalin may have a leading role of in $\mathrm{PG}$.

\section{Dopaminergic Interaction}

In comparison to $5 \mathrm{HT}$ and NA, DA content of neurons in CSF is less. Like NA; DA Can be synthesized from Phenylalanine (Fig. 3). It is believed that the dopaminergic system possibly mediates the positive and negative reward mechanisms and also influences the NA system. Pathological gamblers show signs of arousal [48]. A decrease in the concentration of DA and significant increase in concentration of its metabolites like DOPAC and HVA in the CSF of pathological gamblers have been recorded [48, 49] It is possible that such changes may be responsible for the psychopathology of PG and because of this DOPAC/DA or HVA/DA ratios, i.e. measures of DA release; have been found to be increased. The Dopamine D4 receptor gene which leads to go-slow functioning of the receptor is also linked to PG [48-50].

These biogenic amines in one way or another are involved in PG. A relative balance between various neurotransmitter systems may also contribute to the regulation of impulsive behaviour. An interaction between these three may also exert its effect significantly resulting in decreased serotonin metabolism [51] and increased NA or DA metabolism [52-54]. However various others factors are also responsible for manifestation of impulsive behaviours of

\section{Phenylalanine (Phenylalanine hydroxylase)}

\section{Tyrosine}

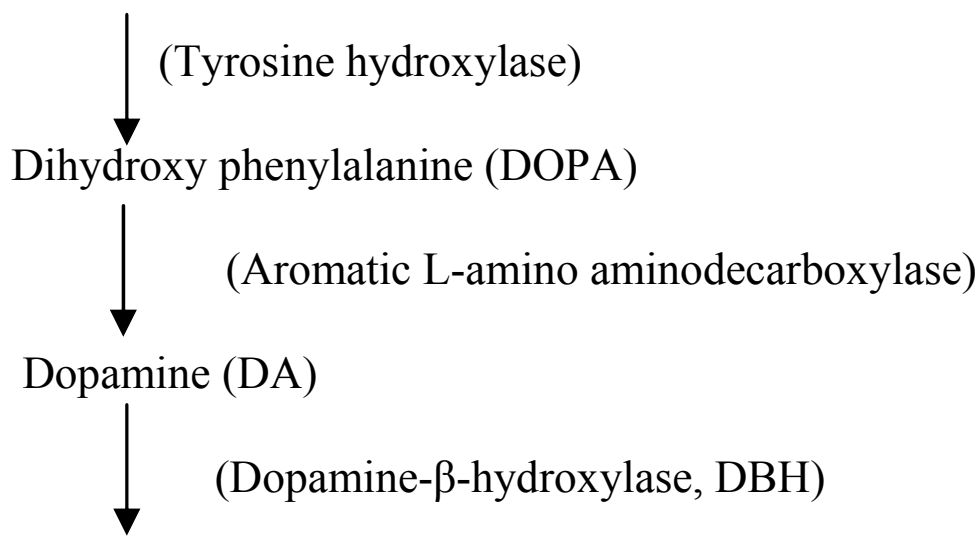

Noradrenalin (NA)

(COMT)

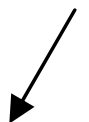

Normetanephrine

(MAO)

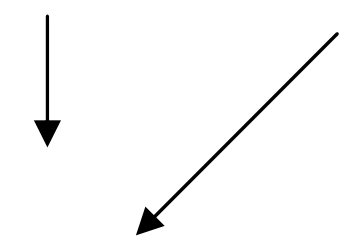

Vanillylmandelic acid (CVMA)
Adrenalin

(MAO)
3,4-Dihydroxyphenylglycol

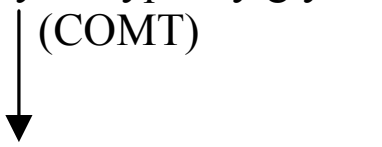

Methoxyhydroxyphenyl glycol (MHPG)

Fig. (2). Regulation of noradrenalin. 


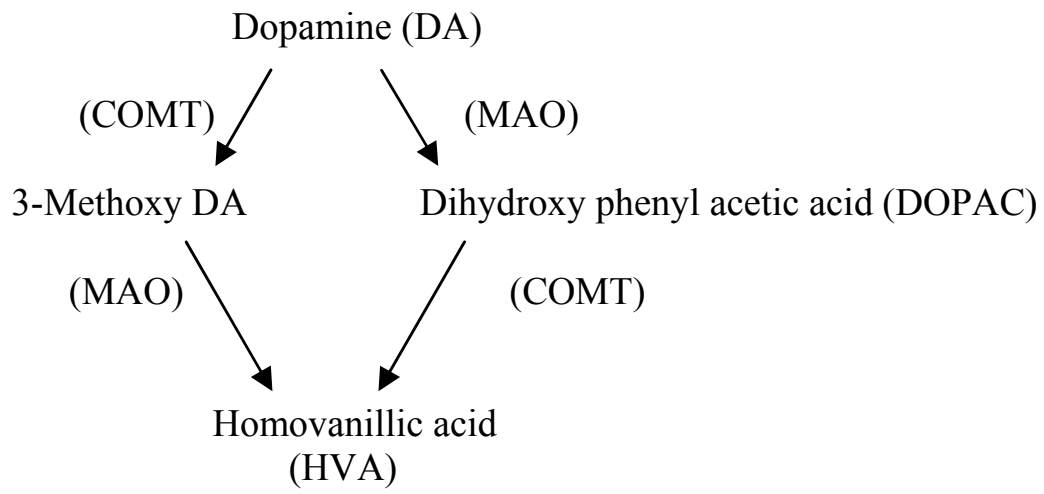

Fig. (3). Regulation of dopamine.

PGS. There is no doubt about the involvement of all of these biogenic amines in cause and maintenance of PG but more studies and regular updates are still required.

\section{CANDIDATE MOLECULES -AMINO ACIDS}

Amino acids play important roles in structure and function of the brain and the nervous system. Amino acid composition of dietary proteins contributes to cerebral functions, whereby some indispensable amino acids present in dietary proteins serve to elaborate neurotransmitters and modulators. Nutrient composition and meal pattern can exert either immediate or long term effects and may be beneficial or detrimental depending upon the nature of the nutrient. Various free amino acids like GABA, aspartic acid, glutamic acid, glycine and taurine play important roles possibly as neurotransmitters or modulators in the CNS and spinal cord. The relative composition of free amino acids in the CNS and CSF depends on a complex equilibrium dependent on transporters at various barriers between blood, CSF and brain as well as on the metabolic processes in the body. The molecules involved in the neurochemistry and subsequently in the neurobiology of pathological gambling are generally synthesized from the amino acids. For example serotonin is generated from tryptophan, and noradrenalin and dopamine from phenylalanine and tyrosine, respectively. Thus an indepth knowledge about such amino acids seems to be essential. Furthermore the literature has provided several leads about their involvement in various disorders, diseases, and deficiencies of the CNS, and also in its corrective measures. Hence it would be logical to have details about such activities especially in pathological gambling.

For the ease of understanding the amino acid section has been divided into to two groups based on having an additional element sulfur, thus, the first part covers all amino acids not containing sulfur, and the second part considers sulfur-containing amino acids.

\section{Amino Acids Not Containing Sulfur}

Under this sub heading first precursors of the 5HT, NA \& $\mathrm{DA}$, i.e. tryptophan, phenyl-alanine and tyrosine will be covered; and then the remaining amino acids will be considered.

\section{Tryptophan}

Tryptophan, a precursor of serotonin (5HT) has been in use since the 1970 s to increase the brain level of serotonin
$[55,56]$. Tryptophan depletion produces marked reduction in plasma tryptophan level; subsequently affecting the brain serotonin synthesis and release. Effects of tryptophan depletion can include mood lowering, memory impairment and an increase in aggression [57]. Tryptophan has a positive effect in depressed patients [58]. Tryptophan depletion produces a relapse of symptoms in patients with depression and panic disorder [59]. But such a situation has been found to be reversed with intravenous tryptophan [60-61], supporting its anti-depression effects. Tryptophan supplementation has been found to have a synergistic effect on Clomipramine therapy [62]. Many other psychiatric and neurological uses of tryptophan have also been advocated [63]. Tryptophan's effect on the human decision making process has been well studied. In one such study 18 healthy volunteers consumed an amino acid drink containing tryptophan and the same number took a drink without tryptophan; and then the effect of gambling was analysed, considering magnitude of expected gains (i.e. reward) vs loss (i.e. punishment), and the possibilities with which these outcomes were delivered. The analysis conformed to the interpretation that tryptophandepleted volunteer's showed reduced discrimination between magnitudes of expected gains associated to different choices. Thus it seems that tryptophan is participating in modulating the process of reward clues directly or through a chain of reactions.

Besides tryptophan its 5 hydroxy derivative (L-5-HTP) also has beneficial effects on sleep terrors. It is believed that it modulates arousal level. Its effect has been well studied in sleep terrors in children [64]. Basically 5-HTP is also an intermediate metabolite of tryptophan produced during serotonin synthesis. The therapeutic use of 5-HTP bypasses its conversion from tryptophan through the enzyme tryptophan hydroxylase, the rate limiting step in serotonin biosynthesis. Supremacy of 5-HTP over tryptophan for antidepression effects has been well recorded [65, 66], however some adverse effects of tryptophan and 5-HTP also exist [67, 68]

In pathological gamblers a higher level of tryptophan has been recorded recently in CSF [8, 69]. In this study a constant higher level of tryptophan was found in all the three fractions of CSF, in comparison to healthy controls. In these studies several factors like intra-spinal pressure, age, tapping time, BMI and some others, affect its concentration [6]. The elevated level of tryptophan in Pathological gamblers may relate to greater participation in the synthesis of serotonin, or 
it is possible that tryptophan may participate directly in stabilizing the situation. Whatever its role may be in $\mathrm{PG}$, a significantly elevated level of tryptophan in PGS warrants an in-depth study.

\section{Phenylalanine}

Phenylalanine is the precursor for two important molecules NA and DA, which are believed to participate in regulation of various behavioural changes. Phenylalanine has been reported also to have the ability to enhance mood. Phenylalanine depletion has been recorded to effect dopamine concentration, possibly by lowering the rate of its biosynthesis. Such observations have been noted also while examining the effects of acute depletion of phenylalanine on mood and Pentagastrin-induced anxiety [70]. Further studies with phenylalanine depletion in patients recovered from depression showed lower concentrations of dopamine, confirming reduced synthesis of dopamine and subsequent mood disturbances [71]. Many studies have been done previously to prove the utility of phenylalanine. In one such study, phenylalanine-depleted and placebo drinks were administered to 20 patients recovered from depression and subjected to cognitive tests as well as selective effects on decision making. It was found that phenylalanine depleted participants bet significantly less. Phenylalanine depletion did not induce relapse in participants, but it did cause patients recovered from depression to show lowered sensitivity to reward in a gambling game [72]. A combination of phenylalanine with a MAO inhibitor may enhance therapeutic potential.

Availability of phenylalanine along with some other neuroactive substances in Pathological gambling has also been examined [73]. Significantly higher concentration of phenylalanine in CSF of Pathological gamblers than in healthy controls has been observed. It is possible that this increase in level of phenylalanine is due to some transport mechanism or for stabilizing the hyper situation; or it simply may be for increased synthesis of NA and DA for their actions, but continued search for convincing answers is required.

\section{Tyrosine}

Tyrosine is an essential amino acid transported from intestine to plasma and then to brain. Tyrosine in fact belongs to a group of 6 neutral amino acids; of which 3 are aromatic; tryptophan, phenylalanine and tyrosine, and the other 3 are valine, isoleucine and leucine (also known as branch chain amino acids). Like phenylalanine tyrosine is also a precursor of NA and DA. Experimental evidences support low availability of tyrosine and reduced DA concentration in CNS [74]. It is believed that higher level of tyrosine will have beneficial effects in regulating mood and behaviour. Experiments provide support for such effects. In a study orally administered $\alpha$-methyl- $\rho$ tyrosine induced relapse in remitted depressed patients. From a mechanistic view this result may indicate $\alpha$-methyl- $\rho$ tyrosine to be competing with endogenous tyrosine, resulting in depletion of ND and DA. Perhaps in the absence of adequate tyrosine there is a possibility of decreased synthesis [75]. Tyrosine therapy has resulted in alleviating depression [76]. Tyrosine depletion in patients recovered from depression has subjective and cognitive effects, having a selective effect on decision making, and lowered sensitivity to reward in a gambling game [70]. In another study on its depletion effect, in response to Pentagastrin-induced acute tyrosine depletion in healthy men, although no significant alterations were observed, the tendency was toward a larger effect on DA than NA [72].

Tyrosine concentration has been monitored in the CSF of healthy controls and Pathological gamblers with significantly increased levels in Pathological gamblers. Although at this junction it is very hard to predict the causes behind this observation, the significant increase must have some logical basis, requiring more experimentation, observation and mechanistic support.

\section{Branch Chain Amino Acids (BCAAS)}

BCAAS are leucine, isoleucine and valine; these are considered to be essential amino acids and are believed to help in improving strength and endurance. In general BCAAS have not shown significant beneficial effects in brain activities; however their supplementations have the short term effect of reducing dopamine levels in healthy emotion-based decision making subjects, characterized by short sightedness, difficulties in resisting short term reward /gain, despite long term negative consequences. Similar effects of BCAAS have been observed on biochemical indices of neurotransmitter function and decision making when also supplemented with tryptophan [77-79].

Measurement of BCAAS content in CSF in Pathological gamblers has shown mixed results [80]; whereas there are increases in concentration of leucine and its isomer isoleucine, no change for valine was observed. The higher concentrations of leucine and isoleucine may be interfering with the physiological actions of core neurotransmitters 5HT, NA and DA; or they may be participating in the process of depletion of the neurotransmitters, thereby aggravating the symptoms associated with deficiencies of these neurotransmitters. It may be also possible that higher concentrations of BCAAS may be helping to ease the situation that arises due to conditions of pathological gambling. The unchanged level of valine seems to indicate its non-participating role in $\mathrm{PG}$.

\section{Excitatory Amino Acids and Others}

A recent study [80] showed that excitatory amino acids such as aspartic and glutamic acids, ornithine, citrulline, and arginine have altered concentrations in CSF of pathological gamblers. Aspartic and glutamic acids are significantly very high. Although these two amino acids are involved in various nervous system actions, their roles in PG have yet to be defined. The higher concentration in PG of these two amino acids must be explained. Amino acids like ornithine, citrulline and arginine belong to the urea cycle and are involved in nitrogen metabolism. Synthesis of any one of the three is interdependent; however significant roles in brain functioning of these amino acids is not yet established. Measurement of the concentrations of these amino acids in CSF of pathological gamblers and healthy controls [80] showed alterations for the Pathological gamblers in comparison to healthy controls. In the case of ornithine a higher level in PGS was found but no significant alteration is recorded for arginine and citrulline. At this stage it is very 
hard to find why there is a marked increase in level of excitatory amino acids like aspartic/glutamic or even ornithine. It seems that an inside view of the mechanism of neural actions in PG has some additional features besides the known involvement of 5HT, NA and DA.

\section{Glycine, and Some More}

Glycine is a non-polar amino acid; it is an inhibitory neurotransmitter in the CNS and in the spinal cord. In contrast to many other amino acids which have been measured recently in CSF of pathological gamblers, glycine content was found to be significantly reduced [80]. Many other amino acids whose concentrations were also measured along with glycine, like threonine and lysine showed almost no change in concentration in CSF of pathological gamblers in comparison to healthy controls. A marked decrease in glycine level in CSF of Pathological gamblers is of concern, and further study is warranted to uncover the mechanism for such a change.

\section{GABA, An Interesting Molecule in PG}

GABA is the chief inhibitory neurotransmitter in the mammalian CNS. It is a surprise to note that depressed gamblers have higher CSF levels of diazepam-binding inhibitors (DBI) than non-depressed gamblers [81]. It is interesting that neurons releasing GABA are known to be a part of an inhibitory system regulating the activity of central noradrenergic neurons [82], thus it is possible that GABA may have some influence on actions of the noradrenergic system. Although very few studies have been done with GABA and pathological gambling, its concentration has been measured in CFS of PGS. In one such study [46] no significant difference in CFS GABA level was recorded for pathological gamblers $v s$ controls or between depressed and non-depressed gamblers; but a significant negative correlation was observed between CSF levels of GABA and the Noradrenalin metabolite 3-methoxy-4-hydroxy phenyl glycol (MHPG) vs Corticotripin releasing hormone (CRH). In general $\mathrm{CRH}$ increases the activity of locus ceruleus NA neurons [83]. Thus a lack of CRH-NA relationship in gamblers suggests a possibility of dysfunction in regulation of the noradrenergic system.

An in-depth study on GABA in pathological gambling has been done by Nordin and Sjodin [8] who found higher concentrations of GABA in CFS of pathological gamblers but disturbed gradients of GABA in healthy controls. In this study GABA concentration was also affected by some physical parameters like age, body mass index (BMI), intraspinal pressure, atmospheric pressure, neuraxis distance, CFS storage time and CSF collection. While most of the factors had no marked effect on GABA level, neuraxis distance contributed to the variance of GABA and thereby increased the magnitude of differences between pathological gamblers and healthy controls. The recent findings of Nordin and Sojdin [8] are just opposite to the earlier finding where no significant difference in CSF GABA level was observed between pathological gamblers and healthy controls. One of the possible explanations advocated by Nordin and Sojdin [8] is that earlier workers analyzed GABA in the $12-32 \mathrm{ml}$ fraction of CSF and the first $12 \mathrm{ml}$ were reserved for another purpose. Thus, it might be possible that there is fractional variation in GABA contents due to the transport mechanism such compounds may be transported caudally in the early morning, Another reason may be due to selection of the participants, where earlier workers have investigated in patients but Nordin and Sojdin intensely invited pathological gamblers though advertisements for such purpose; hence different sets of population might act differently. It is further observed that GABA displayed a disrupted CSF gradient in different fractions.

To further investigate the causes of pathological gambling the role of genetic factors were also examined. In one such study, additional effects of neurotransmitter genes in pathological gambling have been advocated [84]. Thus, it was found that as access to gambling increased there was a corresponding increase in the frequency of addition [of what?] to gambling, which in turn altered the level of neurotransmitters possibly due to genetic contribution. In an another study involving genetic factors and polymorphism, 31 genes involved in 5HT, AD, DA, GABA and some other neurotransmitters were genotyped in 139 pathological gamblers. DA, 5HT and AD genes contributed almost equally to the risk of PG. As GABA is deeply involved in the DA-GABA interaction and there is evidence that GABA transmits, modulates and mediates dopaminergic functions [ 85 ] it is necessary to have detailed studies of such interactions involving GABA which may help in formulating and executing pharmacotherapy of PG.

\section{Sulfur Containing Amino Acids}

Sulfur is not only of economic importance but it is also necessary for life processes. Biological compounds of sulfur are diverse in type and complexity. Sulfur has a coherent biochemistry. Sulfur-containing amino acids are not too many; major representatives are methionine and cysteine. These amino acids have sulfur in its lowest oxidation state $(-2)$ and are needed for protein synthesis. For some reasons sulfur at low oxidation state protects from oxygen toxicity and radiation damage. Reduced sulfur is also utilised in the activation of substrates. Many catalytic molecules and cofactors contain reduced sulfur, including thiamine, biotin, glutathione and many others. The function of all of these molecules depends on the stability and reactivity of reduced sulfur. Thus biochemical actions of sulfur are due to its unique physiochemical properties and because of such unique features amino acids having sulfur have distinct nature and behaviour. Disorders of sulfur metabolism have several effects, in many cases clinically significant; hence metabolic alterations of sulfur, its defects and deficiencies will definitely affect the normal course of action, thereby compelling investigation of the mechanism. Sulfur amino acids have a wide spectrum of actions in the CNS, with high to moderate concentrations in various constituents of the CNS. In pathological gambling concentrations of some sulphur-containing amino acids also have been measured in CSF, like methonine and taurine [7, 8, 73, 80, 86]. Although in the case of methionine no significant difference was observed between pathological gambles and controls, taurine has been found to be of great interest. Initially in an earlier report lower taurine level were measured in CSF of male pathological gamblers than healthy control subjects. To extend further observation on taurine involvement in $\mathrm{PG}$, effects of some other agents on its concentration have been evaluated, namely whether plasma cholesterol and CYP2D6 
phenotype influence taurine concentration [86]. The finding suggests there is a possibility that CYP2D6 may have some influence on taurine level in CSF but no correlation to change in plasma cholesterol was observed. From a mechanistic view during treatment with debrisoquine taurine concentration in the CSF was found to be correlated with the metabolites of Debrisoquine (named DMR. i.e., Debrisoquine metabolic ratio), indicating an influence of the polymorphic CYP2D6 enzyme, which is responsible for hydroxylation of Debrisoquine. In search of more data on taurine involvement in PG [8], pathological gamblers of both sexes (11 male and one female) were subjected to observation and higher levels of taurine were recorded in the CSF of pathological male gamblers, compared to healthy controls. There was a consistently maintained higher concentration gradient of taurine from the first to the third CSF fraction in pathological gamblers compared to healthy controls. In the case of the female pathological gambler the concentration of taurine in CSF was within \pm 2 SD of the corresponding mean value of male pathological gamblers. The level of taurine was as high as two-times the control. Though this latest finding on taurine concentration in PG is of great significance, it produces many questions. Why did the earlier study report lower concentration rather than higher? What is the basis for such opposite findings. It is possible that such differences may be due to the peculiar nature of taurine itself, i.e., its limited ability to cross the blood brain barrier due to its zwitterionic nature, thus affecting its transport to various organs from the intestine. Besides this, techniques and procedure used for lumbar puncture may be responsible for earlier reporting of low concentration of taurine in CSF of PG. In short, there may be many reasons for the diverse results, including increased entry of taurine across the blood brain barrier or reduced elimination from the CSF.

The altered level of various amino acids in PG suggests their a role for them in PG; in summary relative concentrations of amino acids in the CSF in PG can be represented as shown below (Table 1).

Table 1. CSF Amino Acid Concentrations in Pathological Gamblers \& Healthy Male Control; [7, 8, 44, 48, 73, 80]

\begin{tabular}{|c|c|}
\hline Amino Acid & $\begin{array}{c}\text { Over all Difference Between } \\
\text { Gamblers (G) and Control (C) }\end{array}$ \\
\hline \hline Glutamic acid & $\mathrm{G}>\mathrm{C}$ \\
\hline Aspartic acid & $\mathrm{G}>\mathrm{C}$ \\
\hline Phenylalanine & $\mathrm{G}>\mathrm{C}$ \\
\hline Glycine & $\mathrm{G}<\mathrm{C}$ \\
\hline Leucine & $\mathrm{G}<\mathrm{C}$ \\
\hline Isoleucine & $\mathrm{G}=\mathrm{C}$ \\
\hline Lysine & $\mathrm{G}=\mathrm{C}$ \\
\hline Valine & $\mathrm{G}=\mathrm{C}$ \\
\hline Methionine & $\mathrm{G}=\mathrm{C}$ \\
\hline Alanine & $\mathrm{G}=\mathrm{C}$ \\
\hline Citrulline & $\mathrm{G}=\mathrm{C}$ \\
\hline
\end{tabular}

(Table 1) contd.....

\begin{tabular}{|c|c|}
\hline Amino Acid & $\begin{array}{c}\text { Over all Difference Between } \\
\text { Gamblers (G) and Control (C) }\end{array}$ \\
\hline \hline Threonine & $\mathrm{G}=\mathrm{C}$ \\
\hline Serine & $\mathrm{G}=\mathrm{C}$ \\
\hline Aspargine & $\mathrm{G}=\mathrm{C}$ \\
\hline Ornithine & $\mathrm{G}=\mathrm{C}$ \\
\hline Glutamine & $\mathrm{G}=\mathrm{C}$ \\
\hline Arginine & $\mathrm{G}=\mathrm{C}$ \\
\hline Histidine & $\mathrm{G}=\mathrm{C}$ \\
\hline Tryptophan & $\mathrm{G}<\mathrm{C}$ \\
\hline $5-\mathrm{HT}$ & $\mathrm{G}<\mathrm{C}$ \\
\hline Taurine & $\mathrm{G}>\mathrm{C}$ \\
\hline GABA & $\mathrm{G}>\mathrm{C}$ \\
\hline Tyrosine & $\mathrm{G}>\mathrm{C}$ \\
\hline DA & $\mathrm{G}>\mathrm{C}$ \\
\hline NA & $\mathrm{G}=\mathrm{C}$ \\
\hline
\end{tabular}

Many of these amino acids are known to participate in several brain functions and are associated with various diseases /disorders/ dysfunctions. Decreased concentrations of some of these amino acids are of great concern and sometimes the difference becomes a marker of the diseased state. It is believed that replenishment may provide a beneficial effect. In a number of phenomena increased levels of many of these amino acids have been recorded at the site of action. Such increased levels are generally due to active transport and accumulation at some junction in accordance with the need to exert its pharmacological effect for maintaining normalcy and reversing the altered situation. With this hypothesis increased levels of amino acids may be involved in the protection and stabilization mechanism, and use of such amino acids in therapy of PG either as core agents or as supplements/ compliments will be more advantageous over current therapy of PG.

\section{Pharmacotherapy of Pathological Gambling (PG)}

Treatment of pathological gambling is still a subject of much debate. Several schools of thought are running in parallel, based on their ideologies. One such school believes in a hypothesis which has roots in social and behavioural sciences, while the other places PG in under the normal category of sickness. The first school advocates a concept of non-pharmacological treatment using psychoanalysis, psychotherapy, behavioural and cognitive therapy, self help groups, inpatient rehabilitation programs and many others [87-94]. It is possible that some PG suffers may get relief and show signs of improvement using one or more of these therapies; for example, Gamblers Anonymous (GA), a 12 step program may improve some of the associated outcomes of PG [95]. In a similar manner beneficial effects have been associated with treatment of PG with ego-psychology and psychoanalysis approaches [87], but it is still difficult to assess the efficacy of these approaches. Also there is no doubt some success can be achieved with other non pharmacological therapies like cognitive behavioural therapy 
[89] in which the parameter generally used include cognitive restructures, problem solving, social skill training and relapse prevention. Successful cases of behavioural therapy have been observed with aversive therapy and performing electric shock [96], or performing imaginal therapy [97]. In spite of their partial success and occasional beneficial effects such therapies still lack scientific repetition; hence any definite result/outcome warrants consideration of alternative pharmacological treatment.

Despite all of the observations above in reality pharmacotherapy is the only effective tool to manage this chronic, debilitating and largely complex disorder. However it is sad to mention the scarcity of literature on this subject perhaps because of labelling pathological gambling more of a social and psychological problem than a medical illness. In the past very little effort has been devoted to pursuing pharmacotherapy and finding convincing, effective, fast acting, and inexpensive pharmacological agents to treat pathological gambling. Currently agents used for such actions are serotonin reuptake inhibitors (SSRIS), mood stabilizers and opioid antagonists [98-101]. Use of selective serotonin reuptake inhibitors is based on the concept that pathological gambling involves perturbation of the action of selective neurotransmitters in the CNS. As there is the possibility that impulsive control disorder is related to abnormalities in the serotonergic system with alterations in serotonin level, hence SSRIs may have beneficial effects. Thus, it is believed that SSRIs may help to normalize serotonergic functions leading to improved control of impulsive behaviour and other related symptoms. The inhibitors commonly in use are Clomipramine and Fluvoxamine. Many reports exist in the literature on the use of these agents with some success regarding several parameters of pathological gambling such as decreased gambling urge and gambling behaviour [102-104]. Second lines of treatment have involved mood stabilizers. Traditionally mood and gambling are believed to be overlapping; hence, in general significant mood disorders adversely affect control of gambling behaviour. Initially Lithium was in use for mood stabilization $[105,106]$. Though some data are available in the literature, it is unclear how much improvement specific to gambling has been achieved. Another agent Carbamazepine is also in use as a mood stabilizer with improvement in gambling behaviour [104]. The third sets of agents used are opioid antagonists. Since PG rarely exists with a single symptom, so it is possibly blended with reward, addiction, motivation disorders; hence use of opioid antagonist may have some beneficial actions. Thus, Naltrexone has shown signs of improvement in gambling and shopping urges [107]. From a mechanistic view it is believed that opioid antagonists inhibit dopamine (DA) release in the nucleus accumbens, thus modulating the pathways that appear to be involved in the pathogenesis of addictive and related behaviours. With involvement of so many symptoms and causes, it seems that pathological gambling is a composite disorder; hence treating pathological gambling with one agent may not provide very effective results. A single agent may help to alleviate some of the PG effects by reducing some cooccurring symptoms, but for long term beneficial effects alternative approaches need to be considered. The status of biogenic amines and amino acids in PGS should be considered. What is happing to the concentration of various amino acids? Is there a significant alteration in their concentration? If yes, then to what extent are they increased or decreased? The magnitude of such changes requires indepth study. Since significant alteration in concentration of a number of amino acids in the CSF of pathological gamblers has been recently reported, it is logical to think that such amino acids are involved in PG. Various studies on concentration of precursors of known neurotransmitters 5HT, NA, DA have found elevated levels of the precursors; namely, Tryptophan, Phenylalanine and tyrosine in CSF of pathological gamblers. Besides these, reports do exist on higher levels of inhibitory amino acids like GABA and taurine in CSF of pathological gamblers. Recently altered concentration of some other amino acids has been reported in CSF of pathological gamblers, especially glutamic acid, aspartic acid, ornithine and glycine, with elevated concentrations of glutamic acid, aspartic acid and ornithine but decreased level of glycine. At this junction it is very hard to provide genuine and convincing reasons for such increases or decreases, but as glutamic acid and aspartic acid are the only amino acids which have negative charged carboxylic groups separated by one methylene group only, hence it seems that both are behaving similarly like bio isomers. These two amino acids are excitatory neurotransmitters involved in several brain functions, thus they might have some role in PG also. It might be possible due to altered transport that their concentrations have been increased, and the increased concentration may be participating in stabilizing the situation that arises from the cumulative effects of various happenings in PG. Higher level of ornithine may be understood according to a different logic. Orthinine being a urea cycle molecule involved in nitrogen metabolism may be exerting its effect in a little different way. Besides this ornithine is also utilized in arginine synthesis which in turn participates in cell death mechanisms. Thus as of now it is difficult to provide precise reasons for higher ornithine levels in CSF of pathological gamblers. The low level of glycine seems to have some explanation. Chemically glycine is a non polar amino acid and it has no asymmetric carbon atom hence no optical isomerism. Physiologically glycine acts as an inhibitory neurotransmitter with a role in epilepsy. It is used in treatment of manic depression behaviour and hyperactivity. The lower level of glycine in PG may be associated with depression phenomena.

\section{Amino Acids as a Cause for and Source of Pharmacotherapy}

Many amino acids have shown significant alternation in concentration in PG. Thus it is important to consider their involvement in the therapy of PG. For example, tryptophan, whose concentration is higher in PG, may be participating in stabilization through increasing the biosynthesis of more brain serotonin available for action. Besides this serotonin also participates in correcting mood and memory impairment. Since a decreased level of tryptophan is also associated with aggressive behaviour, thus tryptophan supplementation or tryptophan therapy would be useful in PG. Similarly phenylalanine which is a precursor of NA and $\mathrm{DA}$, the core molecules involved in neuro-biology of PG, also has mood enhancing properties. Hence an increased 
level of phenylalanine supports the hypothesis that elevated concentration possibly may be for utilization to enhance the bio-synthesis of NA and DA for exerting their greater influence in PG. As depletion/supplementation of phenylalanine have significant effects on PG test parameters, it may be beneficial to use phenylalanine in PG therapy. A similar case can be made for tyrosine also. Analogous to the other two precursor amino acids, tryptophan and phenylalanine, the concentration of tyrosine has also been reported high in the CSF of pathological gamblers. The higher concentration of all three precursor amino acids provides a basis for their active involvement in the development and/or continuation of PG. Depletion of tyrosine is found to alter the synthesis of ND and DA and also to affect several symptoms in PG; hence use of tyrosine therapy alone or in combination with other therapies hopefully will be beneficial in PG.

Besides altered concentration of precursor amino acids the changed concentrations of other amino acids are also of great significance. The higher level of aspartic acid, glutamic acid and ornithine deserve more attention. Aspartic and glutamic acids are structurally very similar especially the ionic charges and chemical nature where both differ just by a methylene molecule and both are excitatory neurotransmitter with wide spectrum of actions in CNS. It might be possible in one way or other they may be participating in molecular biology of PG. Hence use of such amino acids may give desired effects. Besides higher concentration of excitatory amino acids in PG, a higher concentration of the inhibitory amino acid GABA in the CSF has also been recorded. GABA's role in brain functioning is now more or less established. The higher level of GABA in CSF of pathological gamblers and its link with NA and DA through a number of mechanistic paths suggest a special role in PG. As GABA is in use for treatment of some CNS disorders, it would be a good candidate for complementary/supplementary/ alternative therapy, or even as a primary therapeutic agent.

The importance of non-sulfur-containing amino acids in PG is now beyond doubt, but the same is even true also for sulfur-containing amino acids. The importance of sulfur in life is well known. Several sulfur-containing amino acids actively participate in various life processes from embryonic development to ageing. Some of the sulfur-containing amino acids are of great value; namely, cysteine, methionine and taurine and all three are inter-related. With PG taurine and methionine have been measured, but major studies have been done only for taurine, where effects on plasma cholesterol and CYP2D6 phenotype have been correlated. Since taurine levels recorded in CSF of pathological gamblers are very high, consideration of its involvement is warranted. In several cases where taurine level was recorded to be elevated, the increase reflected greater involvement of taurine in protective mechanisms and in such situations exogenous addition of taurine provided additional benefits $[12,14]$. There is a long list of potential actions for taurine In the CNS, with moderate to significant effects. It is believed that taurine's actions relate to its unique physico-chemical nature and distinguishing characteristics. Since PG is a brain activity where taurine concentration is high, it deserves further attention.

\section{Taurine, a Wonder Molecule}

Taurine is chemically 2 -amino ethane sulfonic acid and it is a $\beta$ amino acid. Though it was discovered about 200 years ago in ox bile, for the first 150 years it was just regarded as a metabolic end product. Taurine came into prominence after the publication of an excellent review by Jacobsen and Smith in 1968 [108] on its physiological actions. Since then understanding of its new roles is emerging and many of the possible roles are being exploited in a positive manner. In almost every mammal taurine is found in a considerably high amount. A normal human of about $70 \mathrm{~kg}$ contains about $0.1 \%$ of its weight as taurine. Taurine has inter-linked physiology, pharmacology, nutrition, biochemistry, and growth and development, and it has beneficial actions in various situations and conditions. For example, benefits have been described for many ailments, from diabetes to heart failure and from liver to eye problems. Because of such actions it has also been patented for many causes and cures. Currently it is also part of formula milk, energy drinks, anti-ageing mixtures, and many more.

In many cases like hypoxia, hyperglycaemia and ischemia free radicals cause neuronal cell damage and death and in almost all such cases taurine has a protective role [9]. The basis for taurine's actions are attributed to its specific physical and chemical nature which includes the presence of a sulfonate group in place of a carboxylate group common to most of the neuro amino acids. Besides this feature, X-ray crystallography shows that taurine is a mono basic acid with unique physical constants in comparison to other neuro active amino acids; e.g., its pka value of 1.5 is more acidic than glycine, aspartic acid, $\beta$ alanine and GABA. Its $\mathrm{pKb}$ value of 8.82 renders it less basic than GABA, $\beta$ alanine, and, glycine. All these distinguishing features reflect the presence of the sulfur atom. Another important characteristic of taurine is that it normally does not participate in peptide formation and protein synthesis, hence it occurs in the free state. Conformational analysis indicates that it has three conformational states of which cyclic is the most stable. There is the possibility of intra-molecular hydrogen bonding in taurine with lower proton affinity of the amino group than in GABA, enabling taurine to penetrate the blood brain barrier albeit at a relatively slow rate. Under different cellular damage conditions generally the level of taurine has been found to elevate; for example in congestive heart failure and spinal cord injury.

In pathological gambling where concentration of taurine has been found to be as high as $150 \%$ of the corresponding level in healthy controls, it is interesting to note that it is high in all the three consecutive CSF fractions obtained from the pathological gamblers compared to the corresponding fractions in healthy male control [109]. Though a well accepted mechanism of neuro protective action of taurine is not yet established, it is believed that taurine exerts its potential not through one agency but by different channels, which include neuro-modulation, osmo-regulation, antioxidation, regulation of calcium ions, and membrane stabilization. In the majority of cases pathological gamblers display coincident addictive behaviours such as alcoholism 


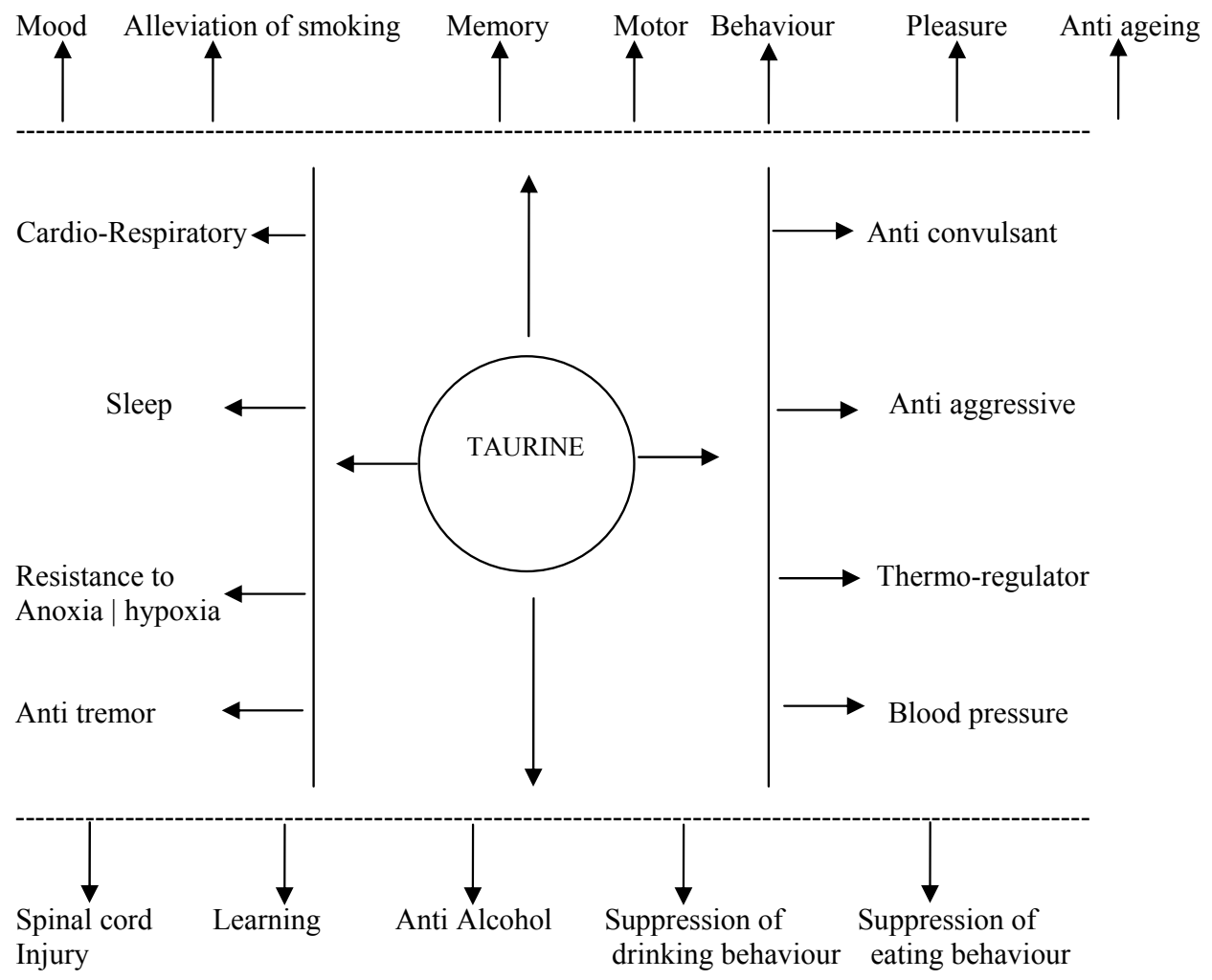

Fig. (4). A holistic view of the central actions of taurine.

and smoking, and sometimes aggressive behaviour also; and of course there is a deep involvement of mood changes. In fact, taurine is in use for many of the cited problems like anti-aggressive treatment [11] anti-convulsant [9], reduction of hang over time, anti-craving effect $[110,111]$ and mood modifier [112]; thus it would perhaps be logical to use taurine in PG (Fig. 4).

\section{Amino Acids as Dietary Requirements for Brain, we are what we Eat?}

In a broad sense individual personality and profile is related to eating habits and nutrients, reflecting a cumulative index of what one eats. Nutrients have significant effects on the structure and functioning of the brain. Nutrients exert such effect even in the womb; therefore healthy food is essential for expectant mothers. Like any other organ brain is elaborated with substances present originally in the diet; in short, vitamins, minerals, amino acids and fatty acids are the core of mental strength and may be responsible for producing cognitive intellectuals. It is only during the past century that the importance's of micronutrients, vitamins, trace elements, and amino acids have gained prominence. Children and adolescents with poor nutritional status are much more exposed to alteration in mental and behavioural functions. This situation can be corrected by dietary measures to a certain extent. Indeed, nutrient composition and meal pattern can exert either immediate or long term effects, beneficial or adverse. Brain diseases especially during ageing generally occur because of failure of protective mechanisms, where essential dietary constituents are lacking, which can exerts actions like anti-oxidants and some other beneficial effects. Radiological studies have also provided a link between micronutrient deficiencies and neuropathology. Many amino acids have direct participation in brain functioning, as neurotransmitters, neuro-modulators, substrates for production of cerebral nitric oxide; and amino acids are also implicated in disease and disorders of the brain like depression, Huntington's disease, Parkinson's disease, as well as in a number of psychiatric disorders. Use of amino acids as dietary supplements for sound health is fast gaining recognition. A large number of amino acids are now part of anti-ageing compositions, mood modifiers, energy drinks and some others are indicated as complements/supplements for additional beneficial actions. Amino acids are now also considered to be a part of alternative medicine therapy, as functional food and medicine.

In $\mathrm{PG}$ concentrations of amino acids like tryptophan, phenylalanine, tyrosine, GABA, taurine and some others have been found to be high. These amino acids in one way or another have protective actions on nerve cells, and also participate in alleviating several symptoms of PG; thus it would be logical to use these amino acids as supplements/compliments to the ongoing drug therapy of the PG patients. From a toxicological viewpoint, the majority of these amino acids are endogenous in nature, so they generally are non toxic. Hence their use will be hopefully less likely to be harmful. 


\section{FUTURE CONSIDERATION-AMINO ACIDS: ANALOGUES \& DERIVATIVES; A HOPE TO COPE}

Use of amino acids will definitely provide beneficial effects on several symptoms associated with pathological gambling. But amino acid therapy has its own limitations. Poor absorption in the gastrointestinal tract and unfavourable ratio between dose administered orally and the corresponding level attained in the CNS are some of the troublesome aspects of amino acid therapy. These problems arise because:

1. Absorption of amino acids from the gastrointestinal tract in to the CNS is limited by active transport mechanisms that have limited capacity.

2. Passive diffusion of most of the amino acids is negligible due to their strong zwitterionic character, highly polar nature and ionized state.

3. Poor absorption is accompanied by high loss through metabolic degradation which occurs both in the gut and in the liver. These losses generally constitute the well known first pass effects.

The majority of the amino acids are affected by the conditions described above, so their significance and potential are limited accordingly. For example, taurine is a potent anticonvulsant agent in an animal epilepsy model when administered intra-cerebroventricularly; it effectively prevents seizures. However, when given orally or administered intraperitoneally, an extremely high dose, typically greater than $3 \mathrm{~g} / \mathrm{day}$ is required to achieve any clinical efficacy. Thus development of pro-drugs of amino acids may afford a way for improving the passive diffusion and also circumventing some of the first pass effects. In the past based on the physical-chemical character of individual amino acids a large number of analogues, homologues, and bio-isosteres having a wide range of CNS actions have been developed; and some are of great therapeutic value. For example, many GABA analogues are useful in therapy of epilepsy [113], S-adenosyl methonine, a derivative of methionine, has anti-depressant efficacy [114]. Acamprosate, a taurine derivative is a well known anti-craving agent [110]. Even in PG; 5-hydroxy tryptophan has clinical utility. In the present scenario where the number of pathological gamblers is sharply increasing there is an urgent need for fast acting analogues of all such amino acids considered to be involved in PG. It might be possible that such analogues can cross the blood brain barrier quickly and easily with much faster rates, and they may hydrolyse /breakdown to provide sufficient amounts of the corresponding amino acids for eliciting physiological effects at the site of action. Hence continued search for analogues of the amino acids would be advantageous for the therapy of pathological gambling and may provide a hope for therapeutic improvements.

\section{CONCLUSION}

This review is perhaps the first of its kind, highlighting the significance of pharmacotherapy and the role of amino acids in pathological gambling; a world wide chronic problem with ascending prevalence. Technically pathological gambling is still not recognized as a unique disease, but it is an impulsive control disorder. In principle several factors are responsible for a behaviour pattern to be called pathological gambling which include after-effects that are more serious than PG itself. There is a large volume of literature on sociological, psychological and behavioural studies of pathological gambling, advocating PG as only a socio-psychological problem and placing medical cause as secondary. While examining the literature one can rightly observe the scarcity of articles and monograms oriented towards the biological basis of $\mathrm{PG}$, and most of what is available deals with the known neurotransmitters 5HT, NA and DA. It is disheartening not to see meaningful literature on the precursors of these neurotransmitters; tryptophan, phenylalanine and tyrosine, which we believe must be involved in the patho-physiological basis of PG; hence this example emphasises the importance of these amino acids in PG, their possible mechanism of action, their metabolites, and their potential superiority as therapeutic agents for PG. Besides these, a large number of other amino acids, which in one way or another participate in various CNS functions from antidepressant to anti-aggressive effects, have been found to have altered content in PG. PG is a disorder with a composite nature, so it might be possible that some other amino acids may be involved in the genesis and development of PG as well as in its alleviation. Alterations in the concentrations of neurotransmitter precursors as well as some other amino acids in the CSF of pathological gamblers may be of great significance. The higher contents of tryptophan, phenylalanine and tyrosine in CSF of pathological gamblers are expected as these amino acids are involved in the biosynthesis of the core molecules 5HT, ND and $\mathrm{AD}$. The finding of higher concentrations of the inhibitory neurotransmitter GABA and the neuro-modulator taurine, and elevated levels of excitatory amino acids like aspartic acid and glutamic acid suggests a role for these amino acids in PG. Considerably elevated content of ornithine in the CSF of pathological gamblers also warrants more studies with this amino acid as it is involved in nitrogen metabolism and arginine synthesis. A decreased level of glycine may be related to its known inhibitory actions. Whatever the reason for the altered concentrations, diversion from the normal state may be either beneficial or detrimental, contributing to the cause or at least serving as a marker for the PG disorder. Accordingly, the significant changes in concentrations of amino acids are likely to have a role in the genesis and development of pathological gambling as well as in its therapy. As more findings of such involvement are reported they will provide strong support for the candidacy of such amino acids as potential lead molecules for development of PG therapy. The majority of the mentioned amino acids are in general non-toxic, endogenous in nature, and part of our food supply and body composition; thus their utilization for therapy will hopefully not have significance adverse effects. In addition to these considerations, currently many amino acids is part of complementary medicine in psychiatry. Several amino acids are being used successfully as nutritional supplements for a number of CNS diseases. Amino acid like aspartic acid whose concentration is significantly elevated in $\mathrm{PG}$, is in use for other CNS actions. Along these lines, if more favourable data on this amino acid in PG is reported, then it may be the one of the potential candidates for PG therapy, but more studies are required. In a similar manner more convincing data and observations on some other amino acids whose 
concentrations are markedly altered in the CSF of pathological gamblers, hopefully will make them eligible for inclusion in PG therapy.

This review has highlighted taurine, an amino acid which contains sulfur and the importance of sulfur in life processes is well known. Because of its multifaceted utility this amino acid is also called "wonder amino acid" along with many other names like "Meta vitamin" and "indispensable molecule." As taurine concentration is recorded very high (almost double) in CSF of pathological gamblers, such a high concentration must be for greater utility and involvement in the process and functioning of PG. Taurine is a beneficial agent in many CNS diseases and disorders, and it is being used alone or in combination with other medicines. Such beneficial effects could be extended to PG also, but extensive basic science and clinical studies are required. In future studies, as taurine is endogenous is nature and non toxic, an in-depth supplementation study may help to formulate the effective doses to alleviate suffering. Currently taurine is a complement/supplement in many alternative food/ medicine compositions; to name a few, these preparations range from anti-ageing to anti-diabetic and mood enhancer agents. Along these lines we can advocate use of taurine and some other candidate amino acids in complementary therapy of PG and also in other psychiatric conditions. It is true that amino acid therapy is a "cool mind therapy," and to observe clinical efficacy requires patience, time and generally higher doses for considerably longer durations. We anticipate such situations because of the unique nature of the amino acids themselves, basically due to their physical-chemical properties. Hence effective, efficient amino acid therapy requires extra attention. To a greater extent, some of the problems associated with amino acid therapy can be overcome by developing analogues, homologues, and derivatives of the amino acids while keeping the core of their effective molecular skeletons intact, with a hope that such analogues will provide sufficient amounts of the amino acids for biological efficacy at the site of action, charting a new avenue for alleviating the suffering from PG.

Much more discussion could be provided on this important topic, but a definite end to this review is necessary. Thus in gist, this presentation covers several amino acids of which some are part of the biosynthesis of core molecules like 5HT, ND and DA, and other amino acids are highlighted whose concentrations are highly altered in PG. As the majority of these amino acids participate in various CNS actions and are generally non toxic, their use as supplements in PG therapy may provide additional benefit, and if in future their analogues are developed to overcome practical pharmacokinetic limitations, then they hopefully would provide a new dimension to the pharmacotherapy of PG. If this therapeutic advance actually occurs, then this review would be a milestone.

\section{REFERENCES}

[1] American Psychiatric Association. Diagnostic and Statistical Manual of Mental Disorders, Fourth Edition. Washington, DC: American Psychiatric Association 1994

[2] Dellósso B, Allen A, Hollander E. Comorbidity issues in the pharmacological treatment of pathological gambling: a critical review. Clin Pract Epidemol Ment Health 2005; 1: 21.
[3] Petry NM, Stinson FS, Grant BF. Comorbidity of DSM-IV pathological gambling and other psychiatric disorders; results from the National Epidemiologic Survey on Alcohol and Related Conditions. J Neural Transm 2005; 66: 564-74.

[4] Blanco C, Ibanez A, Saiz Ruiz J, et al. Epidemiology, pathophysiology and treatment of pathological gambling. CNS Drugs 2000; 13: 397-07.

[5] Lisieur HR, Rosenthal RJ .Pathological gambling: A review of literature. J Gambl Stud 1991; 7: 5-37.

[6] Eklundh T, Nordin C. Intraspinal pressure influences CSF disposition of tryptophan and 5-HIAA. Neuropsychobiology 2001; 44: 84-7.

[7] Nordin C, Sjödin I. Altered CSF taurine function in pathological gamblings. J Psychiatr Res 2006; 40: 473-4.

[8] Nordin C, Sjödin I. CSF monoamine pattern in pathological gamblers and healthy controls. J Psychiatr Res 2006; 40: 454-59.

[9] Huxtable RJ. Taurine in the central nervous system and the mammalian actins of taurine. Prog Neurobiol 1989; 32: 471-33.

[10] Gupta RC, Taurine; insurance of sound health: molecule of the millennium. Ind J Pharmacol 2004; 36: 333.

[11] Mandel P, Gupta RC, Bourguignon JJ, et al. Effects of taurine and taurine analogues on aggressive behavior. Prog Clin Biol Res 1985; 179: 449-58

[12] Gupta RC, Thida W, Bittner S. Taurine analogues: a new class of therapeutic agents: Retrospect and Prospects. Curr Med Chem 2005; 17: 2021-39

[13] Bittner S, Win T, Gupta R. Gamma-L-glutamyltaurine. Amino Acids 2005; 28(4): 343-56.

[14] Gupta RC, Seki Y, Yosida J. Role of taurine in spinal cord injury. Curr Neurovasc Res 2006; 3: 225-35.

[15] Productivity commission. Australia gambling industries: final report. Government press Canberra, Australia 1999.

[16] Gupta R, Derevensky J. Familial and social influences on juvenile behavior. J Gambl Stud 1997; 13: 179-92.

[17] Walker MB, Dickerson MG. The prevalence of problem and pathological gambling: A critical analysis. J Gambl Stud 1996; 12: 233-49.

[18] Shaffer HJ, Hall MN, Vanderbilt MN. Estimating the prevalence of disordered gambling behavior in the United States and Canada: a meta analysis. Boston: Harvard University 1997.

[19] Miller MA, Westermeyer J. Gambling in Minnesota American. J Psychiatry 1996; 153: 845 .

[20] Spunt B, Dupont I, Lesieur H, et al. Pathological gambling and substance misuse: a review of the literature. Subst Use Misuse 1998; 33(13): 2535-60.

[21] Ciarrocchi J. Severity of impairment in dually addicted gamblers. J Gambl Behav 1987; 3: 16-26.

[22] Shaffer HJ, Hall MN. Estimating the pre valence of adolescent gambling disorders: a quantitative synthesis and guide toward standard gambling nomenclature. J Gambling Stud 1996; 12: 19314.

[23] Gupta R. Towards testing a general theory of addictions: An examination of gambling, risk taking and related personality variables in adolescents. Dissertation abstracts international section A: Humanities and Social Science 2000; 60: 4331.

[24] Volberg RA. The prevalence and demographics of pathological gamblers: Implications for public health. Am J Pub Health 1994; 84: $237-41$.

[25] Lesieur HR, Heeineman M. Pathological gambling among youthful multiple substance abuses in a therapeutic community. Br J Addict 1988; 83: 765-1.

[26] McCormick RA, Taber J, Kruedelbach N, et al. Personality profiles of hospitalized pathological gamblers: the California Personality Inventory. J Clin Psychol 1987; 43: 521-7.

[27] Allcock CC, Grace DN. Pathological gamblers are neither impulsive nor sensation-seekers. Aust N Z J Psychiatry 1988; 22: 307-11.

[28] Steel Z, Blaszczynski A. Impulsivity, personality disorders and pathological gambling severity. Addiction 1998; 93: 895-05.

[29] Ciarrocchi J, Richardson R. Profile of compulsive gamblers in treatment: update and comparisons. J Gambl Behav 1989; 5: 53-65.

[30] Livingston J. Compulsive gamblers: observations of action and abstinence. New York Harper Torch Books 1974.

[31] Linden R, Pope H, Jonas J. Pathological gambling and major affective disorder: preliminary findings. J Clin Psychiatry 1986; 41: 201-03. 
[32] American Psychiatric Association. Diagnostic and statistical manual of mental disorders $3^{\text {rd }}$ ed. Washington DC: American Psychiatric Press 1980.

[33] Carrasco JL, Saiz Ruiz J, Monero I, et al. Low platelet MAO activity in pathological gambling. Acta Psychiatr Scand 1994; 90: 427-31.

[34] Rugle LJ, Melamed L. Neuropsychological assessment of attention problems in pathological gamblers, J Nerv Ment Dis 1993; 181: 107-12.

[35] Carlton P, Goldstein L. Physiological determinants of pathological gambling. In Galski T, Ed. The hand book of pathological gambling, 111-135. Springfield, IL: Charles C Thomas 1987.

[36] Anderson G, Brown RIF. Real and laboratory gambling, sensationseeking and arousal: towards a pavlovian component in general theories of gambling and gambling addictions. Br J Psychol 1984; 75: 401-11.

[37] Blaszczynski AP, Winters SW, McCongaghy N. Plasma endorphin levels in pathological gambling. J Gambl Behav 1986; 2: 3-14.

[38] Raylu N, Oei T. Pathological gambling. A comprehensive review. Clin Psychol Rev 2002; 22: 1009-61.

[39] Ibanez A, Blanco C, Perez de Castro I, et al. Genetics of Pathological Gambling. J Gambl Stud 2003; 19: 11-22.

[40] Moreno I, Saiz Ruis J, lopez-ibor JJ. Serotonin and gambling dependence. Hum Psychopharma 1991; 6(Suppl): S9-S12

[41] Balco C, Orensanz-Munoz L, Blanco-Jerez C, et al. Pathological gambling and platelet MAO activity: a psychological study. Am J Psychiatry 1996; 153: 119-21.

[42] DeCaria CM, Hollander E, Grossman R, et al. Diagnosis, neurobiology, and treatment of pathological gambling. J Clin Psychiatry 1996; 57: 80-4.

[43] Levitt P, Pinter JE, Baraekefield XO. Immunocytochemical demonstration of monoamine oxidase $\mathrm{B}$ in brain astrocytes and serotonin neurons. Proc Natl Acad Sci USA 1982; 70: 6385-9.

[44] Nordin C, Eklundin T. Altered CSF 5-HIAA desposition in pathological male gamblers. CNS Spectrum 1998; 4: 25-33.

[45] Roy A, Adinoff B, Roerich L, et al. Pathological gambling. A Psychobiological Study. Arch Gen Psychiatry 1988; 45: 369-73.

[46] Roy A, De Jong J, Linnoila M. Extraversion in pathological gamblers. Correlates with indexes of noradrenergic function. Arch Gen Psychiatry 1989; 46: 679-81.

[47] DeCaria CM, Hollander E, Nora R, et al. Gambling: biological genetic, treatment, government, and gambling concerns neurobiology of pathological gambling, presented at the 150th annual meeting of the American Psychiatric Association, San Diego 1997.

[48] Bergh C, Eklund T, Sodersten P. Altered dopamine function in pathological gambling. Psychol Med 1997; 27: 473-5.

[49] Perez de Castro I, Ibánez A, Saiz-Ruiz J, et al. Genetic association study between pathological gambling and a functional DNA polymorphism at the D4 receptor gene. Pharmacogenetics 1997; 7: 345-48.

[50] Comings DE, Rosenthal R, lesieur HR, et al. A study of the dopamine D2 receptor gene in pathological gambling. Pharmacogenetics 1996; 6: 223-34.

[51] Soubrie P. Reconciling the role of central serotonin neurons in human and animal behavior. Behav Brain Sci 1986; 9: 319-64.

[52] Eichelman B, Thoa NB, Ng KY. Facilitated aggression in the rat following 6 hydroxy dopamine administration. Physiol Behav 1972; 8: 1-3.

[53] Thoa NB, Eichelman B, Richardson JS, et al. 6 Hydroxy dopa depletion of brain nor epinephrine and the facilitation of aggressive behavior. Science 1972; 178: 75-7.

[54] Geyer MA, Segal DS. Shock-induced aggression, opposite effects of intraventricularly infused dopamine and nor epinephrine. Behav Biol 1974: 10: 99-04.

[55] Deniker P, Loo H, Zarifian E. Study of free and total tryptophan in plasma. It value in psychiatry. Encephale 1976; 2: 123-32.

[56] Young SN, Teff KL .Tryptophan availability, 5HT synthesis and 5 Ht function. Prog Neuropsychopharmacol Biol Psychiatry 1989; 13: 373-79.

[57] Van Praag HM .management of depression with serotonine precursors. Biol Psychiatry 1981; 16: 291- 10.

[58] Moller SE, Kirk L, Honore P. Relationship between plasma ratio of tryptophan to competing amino acids and the response to Ltryptophan treatment in endogenously depressed patients. J Affect Disord 1980; 2: 47-59.
[59] Bell C, Abrams J, Nutt D. Tryptophan depletion and its implications for psychiatry. Br J Psychiatry 2001; 178: 399-05.

[60] Neumeister A, Turner EH, Metthews JR, et al. Effects of tryptophan depletion $v s$ catecholamine depletion in patients with seasonal affective disorder in remission with light therapy. Arch Gen Psychiatry 1998; 55: 524-30.

[61] Smith KA, Fairburn CG, Cowen PJ. Relapse of depression after rapid depletion of tryptophan. Lancet 1997; 349: 915-19.

[62] Walider J, Skott A, Carlsson A, et al. Potentiation of the anti depressant action of clomipramine by tryptophan. Arch Gen Psychiatry 1976; 33: 1384-9.

[63] Sandyk R. L-tryptophan in neuropsychiatry disorders: a review. Int J Neuro Sci 1992; 67: 127-44.

[64] Bruni O, Ferri R, Miano S, et al. L-5 Hydroxy tryptophan treatment of sleep terrors in children. Eur J Pediatr 2004; 163: 402-7.

[65] Anonymous. 5 -Hydroxy tryptophan. Altern Med Rev 1998; 3: 224-6.

[66] Byerley WF, Judd LL, Reimherr FW. Grosser BI 5-Hydroxy tryptophan, a review of its antidepressant efficacy and adverse effects. J Clin Psychopharmacol 1987; 17: 127-37.

[67] Steiner W, Fontaine R. Toxic reaction following the combined administration of fluoxetine and L- tryptophan. Five case reports. Biol Psychiatry 1986; 21: 1067-71.

[68] Hertzman PA, Blevins WI, Mayer J, et al. Association of the Eosinophilia-Myalgia Syndrome with the ingestion of tryptophan. N Engl J Med 1990; 322: 869-73.

[69] Grevet EH, Tietzmann MR, Shansis M. Behavioural and acute phenylalanine and tyrosine depletion in healthy male volunteers. Psychopharmacology 2002; 16: 51-5.

[70] Coupland N, Zedkova L, Sanghere G, et al. Response to pentagastrin after acute phenyl alanine and tyrosine depletion in healthy men: a pilot study. J Psychiatr Neuro Sci 2001; 26: 247-51.

[71] Sebelli HC, Fawcett J, Gusovsky F, et al. Clinical studies on the phenyl ethylamine hypothesis of affective disorder: Urine and blood phenyl acetic acid and phenyl alanine dietary supplements. J Clin Psychiatry 1986; 47: 66-70.

[72] Roiser JP, McLean A, Ogilvie AD, et al. The subjective and cognitive effects of acute phenyl alanine and tyrosine depletion in patients recovered from depression. Neuropsychopharmacology 2005; 30: 775-85.

[73] Nordin C, Sjödin I. CSF cholecystokinin, gamma-aminobuturic acid and neuropeptide $\mathrm{Y}$ in pathological gamblers and healthy controls. J Neural Transm 2007; 114(5): 687.

[74] McTavish SFB, Mcpherson MH, Harmer CJ, et al. Antidopaminergic effects of dietary tyrosine depletion in healthy subjects and patients with manic illness. Br J Psychiatry 2001; 179: 356-60.

[75] Neumeister A, Praschak-Rieder N, Hesselmann B, et al. Effects of tryptophan depletion in fully remitted patients with seasonal affective disorder during summer. Psychol Med 1998; 28: 257-64.

[76] Gelenberg AJ, Wojcik JD, Gibson CJ. Tyrosine for depression. J Psychiatr Res 1982; 17: 175-80.

[77] Scerna A, Gijsman HJ, McTavish SF, et al. Effect of branch chain amino acid drink in mania. Br J Psychiatry 2003; 182: 210-13.

[78] Sevy S, Hassoun Y, Bechara A, et al. Emotion-based decision making in healthy subjects: short -term effects of reducing dopamine levels. Psychopharmacology (Berl) 2006; 188: 228-35.

[79] Scarna A, McTavish SF, Cowen PJ, et al. The effects of a branched chain amino acid mixture supplemented with tryptophan on biochemical indices of neurotransmitter function and decision making. Psychopharmacology (Berl) 2005; 179: 761-8.

[80] Nordin C, Gupta RC, Ingemar S. CSF amino acids in Pathological Gamblers and healthy controls. Neuropsychobiology, 2007; 56:152-58.

[81] Miyata M, Mocchetti I, Ferrarese C, et al. Protracted treatment with diazepam increases the turnover of putative endogenous ligands for the benzodiazepine $\beta$-carboline recognition site. Proc Natl Acad Sci USA 1987 ; 84 : 144-8.

[82] Fuxe K, Agnati L, Bolme P, et al. The possible involvement of GABA mechanisms in the action of benzodiazepines on central catecholamine neurons. In Costa E, Greengard P, Eds. Mechanisms of actions of Benzodiazepines. New York Raven Press 1975; p. 75. Valentino R, Foote S. Corticotropin-releasing factor disrupts sensory responses of brain noradrenergic neurons. Neuroendocrinology $1987 ; 45: 28-36$. 
[84] Comings DE, Gade-Andavolu R, Gonzalez N, et al. The additive effect of neurotransmitter genes in pathological gambling. Clin Genet 2001; 60: 107-16.

[85] Menza M. A simple model of the interaction of dopamine, acetylcholine and GABA in movement disorders seen in psychiatry. Med Hypotheses 1988; 27: 285-8.

[86] Nordin C, Eklundh T, Lower CSF. Taurine levels in male pathological gamblers than in healthy controls. Hum Psychopharmacol 1996; 11: 401-03. .

[87] Bergler E. The psychology of gambling. International universities press New York 1957.

[88] Rosenthal RJ .The psychodynamics of pathological gambling: a review of the literature. In Galski T. Ed. The handbook of pathological gambling 1987; pp. 41-70.

[89] Bujold A, Ladouceur R, Sylvain C. Treatment of pathological gamblers: an experimental study. J Behav Ther Exp Psychiatry 1994; $25: 275-82$.

[90] Sylvain C, Ladouceur R, Boisvert JM. Cognitive and behavioral treatment of pathological gambling: A controlled study. J Consult Clin Psychol 1997; 65: 727-32.

[91] Ladouceur R, Mayrand M, Tourigny Y. Risk taking behavior in gamblers and non gamblers during prolonged exposure. J Gambl Behav 1987; 3: 115-22.

[92] Brown R. Arousal and sensation -seeking components in the general explanation of gambling and gambling addictions. Int $\mathrm{J}$ Addict 1986; 21: 1001-16.

[93] McConaghy N, Armstrong MS, Blaszcynski AP, et al. Behavior completion vs. stimulus control in compulsive gambling. Behav Modif 1988; 12: 371-84.

[94] Moody G. Quit compulsive gambling London, England; Thorsons 1900 .

[95] Stewart RM, Brown RI. An outcome study of gamblers anonymous. Br J Psychiatry 1988; 152: 284-8.

[96] Koller KM. Treatment of poker-machine addicts by aversive therapy. Med J Aust 1972; 1; 742-5.

[97] McConaghy N, Armstrong MS, Blaszcynski AP, et al. Controlled comparison of aversive therapy and imaginal desensitization in compulsive gambling. Br J Psychiatry 1983; 142: 366-72.

[98] Kim SW, Grant JE. The psychopharmacology of pathological gambling. Semin Clin Neuropsychiatry 2001; 6: 184-94.

[99] Grant JE, Kim SW. Medication management of pathological gambling. Minn Med 2006; 89: 44-8.
[100] Grant JE, Kim SW, Potenza MN. Advances in the pharmacological treatment of pathological gambling. J Gambl Stud 2003; 19: 85109.

[101] Pallesen S, Molde H, Arnestad HM, et al. Pharmacological treatment of pathological gambling. Tidsskr Nor laegeforen 2006; 126: $1322-4$

[102] Hollander E, Frenkel M, De Caria C, et al. Treatment of pathological gambling with clomipramine. Am J Psychiatry 1992; 149: 710-1.

[103] Hollander E, De Caria CM, Mari E, et al. Short-term single blind fluvoxamine treatment of pathological gambling. Am J Psychiatry 1998; 155: 1781-3

[104] Haller R, Hinterhuber H. Treatment of pathological gambling with carbamazepine. Pharmacopsychiatry 1994; 27: 129.

[105] Moskowitz J. Lithium and lady luck: Use of Lithium carbonate in compulsive gambling. NY State J Med 1980; 80: 785-8.

[106] Moreno I, Saiz Ruiz J. Tratamiento de un caso de ludopatra (juego patologico) con sales de litio. In: Cacabelos R, Ed. Anuario Psiquiatrico. Barcelona: Prous 1991; pp. 249-54.

[107] Kim SW. Opioid antagonists in treatment of impulse-control disorders. J Clin Psychiatry 1998; 59: 159.

[108] Jacobsen JG, Smith LH. Biochemistry and physiology of taurine and taurine derivatives. Physiol Rev 1968; 48: 424-11.

[109] Samuelsson M, Dhal ML, Gupta RC, et al. Taurine in plasma and CSF: A study in healthy male volunteers. Amino Acid 2009; 36: 529-33.

[110] Olive MF. Interaction between taurine and ethanol in central nervous system. Amino Acids 2002; 23: 345-57.

[111] Ward RJ, Kest W, Bruyeer P, et al. Taurine modulates catalase, aldehyde, dehydrogenase and ethanol elimination in rat brain. Alcohol Alcohol 2001; 36: 39-43

[112] Werbuton DM, Bersellini D, Sweeney E. An evaluation of caffeinated taurine drink on mood, memory and information processing in healthy volunteers without caffeine abstinence. Psychopharmacology (Berl) 2001; 158: 322-8

[113] Tan CY, Wainman D, Weaver DF. N-alpha and Beta substituted 3 amino propionic acid acids analogues, design, synthesis and anti seizure activity. Bioorg Med Chem 2003; 11: 113-21.

[114] Tramoni AV, Azorin JM. Therapeutic indications of S-adenosyl methionine in neuropsychiatry. Encephale 1988; 14: 113-8.

(C) R.C. Gupta; Licensee Bentham Open.

This is an open access article licensed under the terms of the Creative Commons Attribution Non-Commercial License (http://creativecommons.org/licenses/by$\mathrm{nc} / 3.0 /$ ) which permits unrestricted, non-commercial use, distribution and reproduction in any medium, provided the work is properly cited. 